\title{
Madera, un Potencial Material Lignocelulósico para la Producción de Biocombustibles en Colombia
}

\author{
Elkin A. Gómez ${ }^{(1)}$, Luis A. Ríos ${ }^{(2)}$ y Juan D. Peña ${ }^{(3)}$ \\ (1) Grupo Procesos Fisicoquímicos Aplicados, Departamento de Ingeniería Química, Universidad \\ de Antioquia, Calle 62 № 52-59, Medellín, Colombia (e-mail: elkinandresgomez@yahoo.com). \\ (2) Grupo Procesos Físicoquímicos Aplicados, Departamento de Ingeniería Química, \\ Universidad de Antioquia, Calle 62 № 52-59, Medellín, Colombia (e-mail: larios@udea.edu.co). \\ (3) Investigación y Desarrollo Negocios Energía, Empresas Públicas de Medellín, E.S.P. Carrera \\ 58 No 42-125, Medellín, Colombia (e-mail: Juan.Pena.Alvarez@epm.com.co).
}

Recibido Mar. 23, 2012; Aceptado May. 16, 2012; Versión final recibida Jul. 13, 2012

\section{Resumen}

Se analiza la disponibilidad del recurso madera en Colombia para evaluar su potencial uso en la producción de etanol carburante. Se analiza la diversidad de especies, las condiciones de plantación y crecimiento de especies arbóreas y la composición química de sus maderas. El análisis indica que casi la mitad del área reforestada con fines comerciales está compuesta por coníferas, entre las que predominan las especies Pinus patula, Pinus caribaea y Eucalyptus grandis. Asimismo se identificaron las especies Pinus patula, Eucalyptus camaldulensis y Eucalyptus grandis como las de más elevado rendimiento y Pinus caribaea, Pinus patula y Eucalyptus camaldulensis como las de más alto contenido de celulosa. Se concluye que dado el gran desarrollo tecnológico y competencia comercial de los productos derivados del pino y eucalipto, Colombia debe aprovechar las ventajas mencionadas y realizar reforestación comercial con estas especies nativas.

Palabras clave: biocombustibles, material lignocelulósico, etanol, biomasa, madera.

\section{Wood, Potencial Lignocellulosic Material for the Production of Biofuels in Colombia}

\begin{abstract}
The availability of woody resources in Colombia is studied for evaluating their potential for the production of ethanol as a fuel. The diversity of species, the planting and growth conditions of trees and the chemical composition of their woods were analyzed. The study shows that about half of the forested area for commercial purposes is formed by conifers, among which Pinus patula, Pinus caribaea and Eucalyptus grandis are the most abundant. Also, the species Pinus patula, Eucalyptus camaldulensis and Eucalyptus grandis showed the highest yield and Pinus caribaea, Pinus patula and Eucalyptus camaldulensis with the highest cellulose content. Given the great technological and highly competitive commercial products derived from pine and eucalyptus, Colombia should take advantage of its situation and promote reforestation with these native species.
\end{abstract}

Keywords: biofuels, lignocellulosic material, ethanol, biomass, wood 


\section{INTRODUCCIÓN}

La crisis energética que se vive actualmente originada en el reconocimiento del carácter no renovable de los combustibles fósiles, ha llevado a una intensa investigación en nuevas tecnologías y en nuevos recursos energéticos. La producción actual de bioetanol se basa en materias primas almidonadas y azucaradas, lo que ha generado un gran debate acerca de su sostenibilidad. En este contexto, el biocombustible producido a partir de biomasa lignocelulósica es una alternativa interesante, ya que las materias primas lignocelulósicas no compiten con los cultivos alimentarios y también son menos costosas que las materias primas agrícolas convencionales. Adicional a esto, la biomasa lignocelulósica es una fuente de energía primaria renovable y adecuada, que puede proporcionar algunas alternativas de combustibles para el transporte como el bioetanol, el biobutanol o el biodiesel en el corto plazo (Hamelinck et al., 2005; Sun \& Cheng, 2002). Es por esta razón que actualmente se encuentran en desarrollo un sinnúmero de tecnologías proyectadas a la producción de bioetanol a partir de este material energético tan abundante. La madera se encuentra entre las materias lignocelulósicas con mayor disponibilidad en el mundo y en los últimos años los principales países productores de madera han centrado su interés en la búsqueda de alternativas para la gestión y la utilización de los residuos derivados de las actividades forestales. Las alternativas de aprovechamiento implementadas hasta el momento se han enfocado fundamentalmente hacia su recuperación energética (Varnero, 2010).

En principio existen dos opciones para el abastecimiento de una cierta demanda de materia prima para la producción de biocombustibles. Una primera opción es adquirir la madera y/o los residuos de los procesos industriales en el mercado. La segunda opción es crear un patrimonio forestal mediante plantaciones para abastecer las necesidades de materia prima.

La biomasa lignocelulósica es la materia prima renovable más abundante, su producción anual se ha estimado 1 x 1010 millones de toneladas alrededor del mundo (Sánchez \& Cardona, 2008). La conversión biológica de las diferentes materias primas lignocelulósicas, como los son los bosques y los residuos agrícolas o los cultivos lignocelulósicos dedicados a la obtención de etanol ofrece numerosos beneficios, pero su desarrollo se ve obstaculizado por los factores económicos y técnicos (Sánchez \& Cardona, 2008). En este sentido, uno de los factores más importantes para reducir el costo de la producción de etanol son los siguientes: utilización eficiente de la materia prima para obtener altos rendimientos de etanol, una alta productividad, alta concentración de etanol en la destilación, la valorización de todos los coproductos y la integración de procesos con el fin de reducir la demanda de energía (Galbe \& Zacchi, 2007).

Por todo esto, el incremento de la seguridad en el abastecimiento energético, la reducción de la dependencia de fuentes de energía de origen fósil, la reducción de las emisiones de gases de efecto invernadero se encuentran entre los principales motivos que han llevado a los diferentes países a impulsar la producción de biocombustibles a partir de los llamados procesos agroenergéticos (Salazar, 2012).

En este documento se analizan la disponibilidad de este recurso en Colombia, su diversidad, las condiciones de plantación y crecimiento de las especies arbóreas más profusas en Colombia y la composición química de sus maderas respecto al contenido celulósico. Con esta información se busca realizar una evaluación integral del recurso maderero colombiano con miras a ser utilizado en la producción de etanol carburante.

\section{CARACTERIZACIÓN ESPECIES MADERERAS EN COLOMBIA}

El agotamiento de algunas especies forestales se debe, entre otras causas, al desconocimiento de la diversidad de especies maderables y sus posibilidades de uso. La incursión en la industria de los biocombustibles ha dado a este importante recurso una aplicación más, una que acarrea beneficios inigualables para el medio ambiente y para la economía mundial. Las investigaciones de los recursos forestales maderables en Colombia se han orientado hacia la determinación de las 
propiedades anatómicas y mecánicas de la madera. La información disponible respecto a la composición química de las maderas de las diferentes especies arbóreas es muy limitada o casi nula.

Dos aplicaciones industriales de los ecosistemas forestales predominan a nivel nacional: La fabricación de pulpa para la industria papelera y el comercio de madera para aserrío. La demanda de madera de estas industrias ha llevado a un marcado decrecimiento del área plantada con especies maderables. Aunque, se han implementado programas de reforestación, éstos no han sido suficientes para mitigar el gran impacto que tienen las industrias antes mencionadas sobre el ecosistema forestal.

\section{Condiciones de plantación especies forestales en Colombia}

Los programas de reforestación que han sido llevados a cabo en Colombia han plantado cerca de la mitad de toda el área reforestada con coníferas, entre las cuales destaca el Pinus patula, seguida de Ciprés (Cupressus $s p$ ) y Pinus tenuifolia. La mayor parte de las plantaciones con fines reforestativos se han ubicado por encima de los $1.600 \mathrm{~m}$ sobre el nivel del mar, áreas en las que también se han establecido plantaciones amplias de Eucaliptos como el Eucalyptus globulus (Cundinamarca y Nariño) y el Eucalyptus grandis (Cauca y Valle). La Tabla 1 muestra las condiciones de plantación más significativas de algunas de las especies forestales más comercializadas en Colombia.

En el piso basal tropical seco, hasta los $2500 \mathrm{~m}$ de altura, se han empleado especies como Tectona grandis, Eucaliptus tereticornis, Eucalyptus camaldulensis, Tabebuia rosea, Bombacopsis quinata y Gmelina arborea. Mientras que en las formaciones húmedas las especies que mayormente se han utilizado son Pinus caribaea, Eucalyptus tereticornis, Pinus oocarpa y Didimopanax morototoni, para Casanare y Vichada en los llanos Orientales. En la Costa Pacífica y otras regiones, se ha utilizado el Cordia alliodora, Jacaranda copaia, Hyeronima chocoensis, Apeiba aspera y Cordia gerascanthus.

Tabla 1: Condiciones de plantación para algunas especies forestales en Colombia.

\begin{tabular}{|c|c|c|c|c|c|}
\hline \multirow{2}{*}{$\begin{array}{c}\text { Nombre común } \\
\text { Especie } \\
\text { (Acosta, 2004) }\end{array}$} & \multicolumn{4}{|c|}{ Condiciones plantación } & \multirow[b]{2}{*}{ Referencia } \\
\hline & $\mathrm{T}\left({ }^{\circ} \mathrm{C}\right)$ & $\mathrm{pH}$ & $\begin{array}{l}\text { Precipitación } \\
\text { anual }(\mathrm{mm})\end{array}$ & $\begin{array}{l}\text { Ubicación } \\
\text { (msnm) }\end{array}$ & \\
\hline Alnus jorullensis & $12-23$ & $4-6$ & $1500-3000$ & $600-3000$ & (CONAFOR, 2010a) \\
\hline Cedrela odorata & $20-32$ & $5-7$ & $1200-3000$ & $0-1200$ & (Barrance, 2004) \\
\hline Cordia alliodora & 23 & $4,5-6,5$ & $600-5000$ & $0-1400$ & (Barrance, 2004) \\
\hline $\begin{array}{c}\text { Cordia } \\
\text { gerascanthus }\end{array}$ & $23-26$ & $6-7$ & $600-2000$ & $0-700$ & (Barrance, 2004) \\
\hline $\begin{array}{l}\text { Cupresus } \\
\text { lusitánica }\end{array}$ & $12-20 a$ & $6,05-7,79 b$ & $1500-2500 a$ & $1400-3300 a$ & $\begin{array}{l}\text { a (Barrance, 2004); } \\
\text { b (Maro, 1991) }\end{array}$ \\
\hline $\begin{array}{c}\text { Eucalyptus } \\
\text { camaldulensis }\end{array}$ & $13-28$ & --- & $250-2500$ & $0-2800$ & (Kropff, 2000) \\
\hline $\begin{array}{l}\text { Eucalyptus } \\
\text { globulus }\end{array}$ & $9-19$ & $5-7$ & $500-2400$ & $2000-3300$ & (Kropff, 2000) \\
\hline $\begin{array}{l}\text { Eucalyptus } \\
\text { grandis }\end{array}$ & $18,8-27,5 a$ & $5-7,5 a$ & $700-4000 b$ & $0-2500 b$ & $\begin{array}{l}\text { a (Duke, 1998a); } \\
\text { b (Kropff, 2000) }\end{array}$ \\
\hline $\begin{array}{l}\text { Eucalyptus } \\
\text { tereticornis }\end{array}$ & $14-27 a$ & $6,5-7,5 b$ & $400-3500 a$ & $0-2350 a$ & $\begin{array}{l}\text { a (Kropff, 2000); } \\
\text { b (Duke, 1998b) }\end{array}$ \\
\hline
\end{tabular}


Tabla 1: (Continuación)

\begin{tabular}{|c|c|c|c|c|c|}
\hline Eucalyptus urophylla & $8-29$ & --- & $1000-1500$ & $500-3000$ & (ICRAF, 1998) \\
\hline Gmelina arborea & $21-28 a$ & $6-8 b$ & $1800-2300 a$ & $0-1400 a$ & $\begin{array}{l}\text { a (Kropff, 2000); } \\
\text { b (Duke, 1998c) }\end{array}$ \\
\hline Pachira quinata & $20-27$ & $5,5-7,5$ & $800-2000$ & $0-800$ & (Barrance, 2004) \\
\hline Pinus caribaea & $20-27 a$ & $5-5,5 b$ & $600-1800 a$ & $0-800 a$ & $\begin{array}{l}\text { a (Barrance, 2004); } \\
\text { b (Kropff, 2000) }\end{array}$ \\
\hline Pinus kesiya & $14-23$ & --- & $700-2200$ & $600-1800$ & (Kropff, 2000) \\
\hline Pinus maximinoi & 19 & $4,2-6,5$ & $1000-2000$ & $600-2400$ & $\begin{array}{l}\text { (CONAFOR, } \\
\text { 2010b) }\end{array}$ \\
\hline Pinus oocarpa & $13-23 a$ & $5-6 b$ & $700-1500 b$ & $600-2000 a$ & $\begin{array}{l}\text { a (Barrance, 2004); } \\
\text { b (Kropff, 2000) }\end{array}$ \\
\hline $\begin{array}{c}\text { Pino } \\
\text { Pinus patula }\end{array}$ & $9-23 a$ & $4,5-6 b$ & $1000-2200 a$ & $1000-3300 a$ & $\begin{array}{l}\text { a (Kropff, 2000); } \\
\text { b (AGROSOFT, } \\
2000)\end{array}$ \\
\hline Pinus radiata & $8-18$ & --- & $650-1600$ & $1500-3000$ & (Kropff, 2000) \\
\hline Pinus tecunumanii & $14-25$ & --- & $790-2200$ & $440-2800$ & (Barrance, 2004) \\
\hline $\begin{array}{l}\text { Schizolobium } \\
\text { parahibum }\end{array}$ & $19-23$ & $5,5-7$ & $1100-2500$ & $0-650$ & (Barrance, 2004) \\
\hline Tabebuia roseae & $19-27$ & $5,1-6,5$ & $1200-2500$ & $0-1300$ & (Barrance, 2004) \\
\hline Tectona grandis & $>18$ & --- & $1000-2500$ & $0-1200$ & (Kropff, 2000) \\
\hline
\end{tabular}

Dado que en los recientes gobiernos no se han ofrecido políticas que estimulen el aprovechamiento sostenible de los diferentes bosques naturales del país y debido a la falta de aplicación de las leyes por parte de las autoridades ambientales en la explotación tanto informal como legal de los bosques y en la comercialización de la madera (no se siguen los planes forestales de manejo ni se tramitan los permisos), se presenta un deterioro heterogéneo en toda la cobertura forestal colombiana. Los bosques naturales continúan siendo la principal fuente de abastecimiento de la madera utilizada en el país. Según diversas estimaciones, el volumen de madera suministrado por el bosque natural constituye un $70 \%$ a $80 \%$ de la oferta nacional. Por otro lado, la mayoría de los aprovechamientos legales conllevan asimismo a efectos similares sobre el bosque, debido a que la mayoría de los permisos otorgados por la autoridad son en pequeñas áreas, hay en consecuencia una corta duración del proceso y el esquema no es propicio para buscar el manejo sostenible del recurso. Sin embargo, actualmente tiene vigencia un estímulo gubernamental que busca privilegiar la siembra con fines protectivos o comerciales de algunas especies forestales (Alnus jorullensis, Bombacopsis quinata, Cedrela odorata, Schizolobium parahibum, Tabebuia rosea, Eucalyptus grandis, Eucalyptus globulus, Pinus patula, Gmelina arborea entre otras). La resolución 711 del 31 de octubre de 1994 con algunas modificaciones determina las especies beneficiarias del Certificado de Incentivo Forestal (CIF), certificado que se traduce en un aporte directo en dinero, como un reconocimiento del Estado a las actividades positivas desarrolladas en reforestación, que consiste en un bonificación en efectivo de los costos de siembra de plantaciones forestales con fines protectores y productores en terrenos de aptitud forestal, del $50 \%$ si se plantan especies introducidas y un $75 \%$ si se plantan especies nativas. Por los costos totales netos de manejo del segundo hasta el quinto año se reconoce hasta un $50 \%$ para ambos casos. Se ha adicionado un párrafo describiendo este privilegio (Minagricultura, 2012) 


\section{Especies más utilizadas en la reforestación comercial}

Según el Ministerio del Medio Ambiente, el área total de plantaciones forestales comerciales hasta el 2002 era igual a 174.121 ha. Sin embargo el Plan Nacional de desarrollo Forestal indica que en el país existen aproximadamente veinticinco millones de hectáreas de tierras aprovechables, de los cuales tres millones de hectáreas que se encuentran subutilizadas por el sector agropecuario de manera poco sostenible, podrían ser incorporadas a la base forestal productiva del país a corto plazo. De la zonificación de áreas para plantaciones comerciales se concluye que existen en el territorio colombiano unos 4,5 millones de hectáreas con aptitud forestal comercial sin restricciones y unos 11,5 millones de hectáreas con aptitud forestal comercial y restricciones menores, en los que las restricciones se refieren a factores edafológicos que pueden manejarse con tecnologías y técnicas apropiadas. Es decir, existen en el país aproximadamente 16 millones de hectáreas susceptibles a la reforestación comercial, número de hectáreas muy superior a las 174.241 ha de existencias en plantaciones comerciales, lo que indica un subdesarrollo de este sector económico frente a su potencial. Sin embargo los datos reportados no dejan de estar sesgados debido a aspectos técnicos como las limitaciones en la escala de análisis de las imágenes satelitales y a que los regímenes de permisos de aprovechamiento forestal describen tamaños de explotación hasta de 15 ha, muy pequeños para ser registrados.

Actualmente se encuentra en desarrollo, y desde el 2003, un plan de Inventario Forestal Nacional (IFN) en fase de implementación, coordinado por el Instituto de Hidrología Meteorología y Estudios Ambientales (IDEAM), el Ministerio de Ambiente y Desarrollo Sostenible (MAVDT) y el Departamento Nacional de Estadísticas (DANE). En la Tabla 2 se muestra el área plantada con especies forestales comerciales en Colombia. Según los registros de plantaciones comerciales, alrededor del $73 \%$ del área plantada son con especies introducidas como pinos y eucaliptos; sólo el $9 \%$ lo está con especies nativas.

Tabla 2: Especies más utilizadas en la reforestación comercial (SITEP, 1999)

\begin{tabular}{cccc}
\hline \hline Nombre Científico & Origen & Área (Ha) & $\%$ \\
\hline \hline Alnus jorullensis & Nativa & 888.02 & 0.61 \\
Cederla odorata & Nativa & 374.9 & 0.26 \\
Cordia alliodora & Nativa & 925.7 & 0.64 \\
Cordia gerascanthus & Nativa & 168.3 & 0.12 \\
Cupresus lusitánica & Exótica & 9982.2 & 6.85 \\
Especies varias & Exótica & 6802.37 & 4.64 \\
Eucalyptus camaldulensis & Exótica & 787 & 0.54 \\
Eucalyptus globulus & Exótica & 5024.2 & 3.45 \\
Eucalyptus grandis & Exótica & 15265.9 & 10.47 \\
Eucalyptus sp. & Exótica & 2155.49 & 1.48 \\
Eucalyptus tereticornis & Exótica & 3403.4 & 2.33 \\
Eucalyptus urograndis & Exótica & 272.5 & 0.19 \\
Eucalyptus urophylla & Exótica & 214.3 & 0.15 \\
Gmelina arbórea & Exótica & 5083.8 & 3.49 \\
Pachira quinata & Nativa & 6394 & 4.39 \\
Pinus caribaea & Exótica & 10365.7 & 7.11 \\
Pinus kesiya & Exótica & 3811.3 & 2.61 \\
Pinus maximinoi & Exótica & 315.5 & 0.22 \\
Pinus oocarpa & Exótica & 7998.68 & 5.49 \\
\hline \hline & & &
\end{tabular}


Tabla 2: (Continuación)

\begin{tabular}{cccc}
\hline \hline Pinus patula & Exótica & 53197.64 & 36.5 \\
Pinus radiata & Exótica & 143.8 & 0.1 \\
Pinus tecnuifolia & Exótica & 169 & 0.12 \\
Pinus tecunumanii & Exótica & 4277.7 & 2.93 \\
Schizolobium parahibum & Nativa & 247.8 & 0.17 \\
Tabebuia roseae & Nativa & 3988.3 & 2.74 \\
Tectona grandis & Exótica & 3501.85 & 2.4 \\
\hline \hline
\end{tabular}

En cuanto a la propiedad del sector forestal comercial, un gran porcentaje del área plantada es propiedad de unas pocas empresas privadas. La gran participación de la empresa privada en el sector forestal se decidió como una estrategia para aprovechar los incentivos tributarios provistos por el gobierno y para garantizar la propiedad de la tierra ante programas de reforma agraria, en otros casos, empresas que aprovechaban la madera del bosque natural para su actividad industrial, decidieron recurrir a las plantaciones para generar su materia prima como forma de superar las dificultades en el manejo de las concesiones forestales y en respuesta a presiones internas y externas que dificultaban el aprovechamiento industrial del bosque natural.

La contribución de las plantaciones al suministro de madera industrial en el país, se estima entre el $12 \%$ y el $20 \%$ del volumen total.

Aunque los esfuerzos en el campo del mejoramiento forestal mediante prácticas de selección de especímenes se iniciaron desde el decenio del sesenta con especies como Eucalyptus globulus, Colombia continúa sin tener programas de mejoramiento bien establecidos debido al carácter indeterminado del origen de las semillas que se emplean en los programas que se han adelantado y a la carencia de un plan de mejoramiento genético de las semillas a emplear. Una prueba de ello es el bajo desarrollo obtenido con las plantaciones realizadas (Trujillo, 2005). Más recientemente el INDERENA inició proyectos de mejoramiento con las especies Eucalyptus globulus y Cupressus Iusitanica en Cundinamarca y Antioquia, pero la desaparición de la entidad hizo que concluyeran las investigaciones adelantadas. Entonces el Ministerio de Agricultura y Desarrollo Rural inició en 1996 junto al CONIF el programa de Investigación en Semillas de especies Forestales Nativas (INSEFOR) con miras a ofrecer semillas mejoradas genéticamente (Trujillo, 2005). Un resultado del programa fue el establecimiento de algunos huertos semilleros de Tectona grandis, Alnus acuminata, Tabebuia roseae y Cordia alliodora. Algunos avances en el campo del mejoramiento genético forestal han sido impulsados por empresas como Cartón de Colombia, Pizano S.A. y Refocosta en aras de aumentar la productividad forestal por unidad de área. Claro ejemplo de este esfuerzo empresarial es Smurfit Cartón de Colombia, que ofrece semillas mejoradas de las especies Pinus patula, Eucalyptus grandis, Eucalyptus urograndis y Cupressus Iusitanica, pero que no desarrolla procesos de mejoramiento con especies locales, lo que acarrea serias limitaciones de adaptabilidad frente a las necesidades forestales del país (Trujillo, 2005).

Dado que las fuentes semilleras colombianas se han desarrollado levemente por el aprovechamiento primario de la madera en el negocio de la exportación (las semillas se extraen de los árboles remanentes de calidad baja que al entrecruzarse originan individuos forestales aún más inferiores en las características deseadas), algunas plantaciones forestales comerciales emplean semillas mejoradas producidas en Costa Rica y comercializadas por el Centro Agronómico Tropical de Investigación y Enseñanza (CATIE), organización de la OEA adscrita al Instituto Interamericano de Cooperación para la Agricultura (IICA). Las semillas adquiridas en el CATIE han probado tener buenos resultados en Colombia tanto en el aspecto genético como en el caso de la eficiencia de la semilla como tal (Trujillo, 2005), es por esto que la información aportada por dicha entidad costarricense deberá ser tenida en cuenta en esta investigación. 


\section{Rendimiento de las plantaciones, ubicación y turnos de crecimiento}

El recurso forestal puede analizarse desde el punto de vista del rendimiento de sus plantaciones y desde sus turnos de crecimiento. El rendimiento volumétrico de madera por unidad de área y de tiempo es una medida de productibilidad de las diferentes especies forestales $y$, al usarse en conjunto con la periodicidad de los cultivos, es un factor decisivo a la hora de seleccionar una fuente lignocelulósica como la más apta para ser empleada en la producción de biocombustibles. Asimismo, la distribución geográfica de los recursos maderables tiene una marcada influencia en la economía del procesamiento de los materiales lignocelulósicos puesto que el transporte de la biomasa aumenta considerablemente los costos de procesamiento de toda materia prima.

En este sentido Colombia es un país con marcadas ventajas. En Colombia las especies forestales tardan menor tiempo para crecer y ser productivas, de lo que tardarían en otros países. En el caso de eucaliptos se pueden lograr rendimientos de hasta $30 \mathrm{~m}^{3} /($ ha $\times a \tilde{n} o)$ con un turno de 8 años (Proexport, 2012). En cuanto a aptitud forestal la Corporación Nacional de Investigación y Fomento Forestal (CONIF, 2009) realizó un estudio de zonificación por aptitud forestal teniendo en cuenta áreas abiertas, pastizales y suelos que actualmente tienen otros usos (excluye terrenos cubiertos por bosques). Este estudio estimó que en Colombia existen 17 millones de has con aptitud forestal (2008), de las cuales 5,1 millones son tierras sin restricciones, es decir que no requieren ningún tipo de adecuaciones de suelos para el cultivo y desarrollo de proyectos forestales y 12,1 millones tienen restricciones menores.

Los programas de plantación forestal en Colombia aún se encuentran en sus inicios, prueba de ello es la pobre gestión en el sector de la empresa privada privado, la escasa disponibilidad de información oficial sobre el cultivo de las especies de mayor utilización en el país y el apoyo débil y confuso del estado a la reforestación. A la hora de llevar a cabo planes de reforestación en Colombia, se han cometido grandes errores al concebir proyectos, escoger sitios de cultivo, seleccionar especies y manejar las plantaciones, sin una clara orientación sobre la utilización de la materia prima. Toda esta serie de factores ha conducido a que una significativa extensión de plantaciones presente crecimientos por debajo de los estimados, altas tasas de mortalidad forestal por ataques microbianos y calidad inferior de los productos. Entre las actividades reforestadoras llevadas a cabo en territorio colombiano destacan las que se han implementado con especies de coníferas y latifoliadas, con diferentes objetivos económicos e industriales y realizadas por la empresa privada:

\section{Coníferas}

En términos generales, el manejo de las plantaciones de coníferas ha seguido los lineamientos propuestos por la empresa privada dedicada exclusivamente a la producción de pulpa y puesto que esta empresa privada no vislumbró una posible diversificación industrial, el comercio actual está restringido a la producción de papel, con pocas excepciones enfocadas a producir madera de aserrío o madera para inmunización, como productos primarios. Adicional a esto, un mercado irregular y muy confuso de la madera como producto primario, dado el gran número de pequeños comerciantes, ha llevado a un vacío técnico en el cultivo de especies forestales. Sumado a esto se encuentra la gran variabilidad de precios en la industria forestal debida principalmente a factores como distancia de transporte de insumos y falta de mecanización, atribuidos al carácter agreste de las condiciones de suelo y flora colombianas.

Antioquia, Casanare, Cundinamarca y Vichada se encuentran entre los departamentos que más han experimentado la reforestación con coníferas con Pinus caribaea, Pinus oocarpa Pinus patula y Pinus radiata como especies predominantes. Estas especies de pino han probado tener rendimientos verdaderamente elevados (Tabla 3) con rangos comprendidos entre los 20 y los 30 $\mathrm{m}^{3} /($ ha $\times a \tilde{n} o)$. En los Llanos Orientales y la Costa Atlántica se espera la aplicación de sistemas silviculturales diferentes a los de la zona andina, debido a la influencia de la mecanización en las 
labores de establecimiento, manejo y aprovechamiento de las plantaciones y a los usos eventualmente diferentes de la madera (Proexport, 2012)

\section{Latifoliadas}

En latifoliadas Colombia después de Brasil registra los mayores rendimientos con 30 $m^{3} /(h a \times a \tilde{n} o$ ) en un turno de 7 años (Proexport, 2012). El grueso de las plantaciones de latifoliadas de turno corto lo conforma el género Eucaliptus. En la Sabana de Bogotá, donde el Eucalyptus glóbulus tiene una alta demanda para construcción y para inmunización, las plantaciones se inician con una densidad alta, la cual se va reduciendo a medida que se aprovechan ejemplares comercializables. En el Valle del Cauca, Cauca, Antioquia y Costa Atlántica, se utilizan las especies Eucalyptus grandis, Eucalyptus tereticornis y Eucalyptus camaldulensis, en turnos entre 6 y 8 años, para producir materia prima para pulpa o tableros de fibra y partículas. En la actualidad el Eucalipto es considerado como especie marginal para la industria del aserrío o de tableros contrachapados en Colombia, razón por la cual no se ha pensado en la adopción de sistemas silviculturales con turnos de mediana duración, como sucede en Chile y Argentina, países productores de chapa, madera aserrada y muebles de Eucalyptus globulus y Eucalyptus grandis. En la Costa Atlántica se impulsó la investigación genética para la plantación de Gmelina arborea y Bombacopsis quinata, para la producción de tableros. Especies como E. grandis, E. camaldulensis, y Gmelina arbórea han dado buenos rendimientos de 25, 35, y $25 \mathrm{~m}^{3} /($ ha $\times a \tilde{n} o)$, respectivamente. Rendimientos similarmente altos se listan en la tabla 3 para otras latifoliadas de turno corto (Proexport, 2012).

Las especies que podrían tener un futuro importante en la silvicultura tropical de plantaciones densas en Colombia son la Teca (Tectona grandis) y el Abarco (Cariniana pyriformis). Las demás especies plantadas con fines de producción de maderas finas, tales como el Roble (Tabebuia rosea), la Ceiba tolúa, Canalete (Cordia gerascathus), Laurel (Cordia alliodora), Achapo (Cedrelinga sp.) requieren turnos de menor duración.

Tabla 3: Producción para especies forestales en Colombia

\begin{tabular}{|c|c|c|c|c|c|}
\hline $\begin{array}{l}\text { Especie } \\
\text { Forestal }\end{array}$ & $\begin{array}{l}\text { Turnos } \\
\text { (años) }\end{array}$ & $\begin{array}{l}\text { Densidad } \\
\left(\mathrm{g} / \mathrm{cm}^{3}\right)\end{array}$ & $\begin{array}{c}\text { Rendimiento } \\
\left(m^{3} /(h a \times a \tilde{n} o)\right)\end{array}$ & $\begin{array}{c}\text { Presencia en } \\
\text { Colombia }\end{array}$ & Referencias \\
\hline $\begin{array}{l}\text { Alnus } \\
\text { jorullensis }\end{array}$ & $20 a$ & $0,4-0,6 a$ & $10-20 a$ & $\begin{array}{l}\text { Antioquia, Boyacá, } \\
\text { Caldas, } \\
\text { Cundinamarca, } \\
\text { Risaralda b }\end{array}$ & $\begin{array}{l}\text { a (Barrance, 2004); } \\
\text { b (Escobar, 1993) }\end{array}$ \\
\hline $\begin{array}{l}\text { Cedrela } \\
\text { odorata }\end{array}$ & $18-25 a$ & $0,44 b$ & $11-22 c$ & $\begin{array}{l}\text { Antioquia, } \\
\text { Caquetá, Cauca, } \\
\text { Valle del Cauca }\end{array}$ & $\begin{array}{l}\text { a (Barrance, 2004); b } \\
\text { (Wishnie, 2007); } \\
\text { c (Escobar, 1993) }\end{array}$ \\
\hline $\begin{array}{l}\text { Cordia } \\
\text { alliodora }\end{array}$ & $20-30 a$ & $0,46 b$ & $5-20 c$ & Antioquia c & $\begin{array}{l}\text { a (Barrance, 2004); b } \\
\text { (Wishnie, 2007); } \\
\text { c (Escobar, 1993) }\end{array}$ \\
\hline $\begin{array}{l}\text { Cupresus } \\
\text { lusitánica }\end{array}$ & --- & 0,44 & 18 & & (Escobar, 1993) \\
\hline $\begin{array}{l}\text { Eucalyptus } \\
\text { camaldulensis }\end{array}$ & $6-8 a$ & $0,815 b$ & $20-35 c$ & $\begin{array}{l}\text { Antioquia, Cauca, } \\
\text { Valle del Cauca a }\end{array}$ & $\begin{array}{l}\text { a (Acosta, 2004); } \\
\text { b (Cinco, 2005); } \\
\text { c (Kropff, 2000) }\end{array}$ \\
\hline $\begin{array}{l}\text { Eucalyptus } \\
\text { globulus }\end{array}$ & $10-15 a$ & $0,7 b$ & $15-20 a$ & $\begin{array}{l}\text { Antioquia, Boyacá, } \\
\text { Caldas, } \\
\text { Cundinamarca, } \\
\text { Nariño b, c } \\
\end{array}$ & $\begin{array}{l}\text { a (Skolmen, 2004); } \\
\text { b (Escobar, 1993); } \\
\text { c (Acosta, 2004) }\end{array}$ \\
\hline
\end{tabular}


Tabla 3: (Continuación)

\begin{tabular}{|c|c|c|c|c|c|}
\hline $\begin{array}{l}\text { Eucalyptus } \\
\text { grandis }\end{array}$ & $6-8 a$ & $0,45 b$ & $25 b$ & $\begin{array}{l}\text { Antioquia, } \\
\text { Cauca, Valle del } \\
\text { Cauca a }\end{array}$ & $\begin{array}{l}\text { a (Acosta, 2004); } \\
\text { b (Meskimen, } \\
2004)\end{array}$ \\
\hline $\begin{array}{l}\text { Eucalyptus } \\
\text { tereticornis }\end{array}$ & $6-8 a$ & $\begin{array}{l}0,660- \\
1,060 b\end{array}$ & $18 \mathrm{c}$ & $\begin{array}{l}\text { Antioquia, } \\
\text { Casanare, } \\
\text { Cauca, Valle del } \\
\text { Cauca, Vichada } \\
\text { d }\end{array}$ & $\begin{array}{l}\text { a (Acosta, 2004); } \\
\text { b (Kropff, 2000); } \\
\text { c (Duke, 1998b); } \\
\text { d (Acosta, 2004) }\end{array}$ \\
\hline $\begin{array}{l}\text { Eucalyptus } \\
\text { urophylla }\end{array}$ & --- & $0,55 a$ & $28-30 b$ & --- & $\begin{array}{l}\text { a (Naumann, } \\
\text { 2008); } \\
\text { b (Gifoni, 2004) }\end{array}$ \\
\hline $\begin{array}{l}\text { Gmelina } \\
\text { arborea }\end{array}$ & $\begin{array}{l}10- \\
14 a\end{array}$ & $0,40-0,51 b$ & $20-25 a$ & --- & $\begin{array}{l}\text { a (CONIF, 2009); } \\
\text { b (Kropff, 2000) }\end{array}$ \\
\hline $\begin{array}{l}\text { Pachira } \\
\text { quinata }\end{array}$ & $\begin{array}{l}24- \\
29 a\end{array}$ & $0,39 b$ & $10-15 a$ & --- & $\begin{array}{l}\text { a (Barrance, 2004); } \\
\text { b (Escobar, 1993) }\end{array}$ \\
\hline $\begin{array}{l}\text { Pinus } \\
\text { caribaea }\end{array}$ & --- & $0,39-0,40 a$ & $10-40 b$ & $\begin{array}{l}\text { Casanare, } \\
\text { Vichada c }\end{array}$ & $\begin{array}{l}\text { a (Zamora, 2004); } \\
\text { b (Kropff, 2000); } \\
\text { c (Acosta, 2004) }\end{array}$ \\
\hline Pinus kesiya & --- & $0,40-0,75$ & $10-30$ & --- & (Kropff, 2000) \\
\hline $\begin{array}{l}\text { Pinus } \\
\text { maximinoi }\end{array}$ & --- & $0,41 a$ & $10-23 a$ & --- & (Fonseca, 2006) \\
\hline $\begin{array}{l}\text { Pinus } \\
\text { oocarpa }\end{array}$ & $\begin{array}{l}23- \\
30 a\end{array}$ & $0,42-0,6 b$ & $10-40 a$ & $\begin{array}{l}\text { Casanare, } \\
\text { Vichada c }\end{array}$ & $\begin{array}{l}\text { a (Kropff, 2000); } \\
\text { b (Barrance, 2004); } \\
\text { c (Acosta, 2004) }\end{array}$ \\
\hline Pinus patula & $30 a$ & $0,43 b$ & $27 a$ & $\begin{array}{l}\text { Antioquia, } \\
\text { Cauca, } \\
\text { Cundinamarca, } \\
\text { Santanderes, } \\
\text { Valle del Cauca } \\
\text { b }\end{array}$ & $\begin{array}{l}\text { a (Gillespie, 2004); } \\
\text { b (Escobar, 1993) }\end{array}$ \\
\hline Pinus radiata & $\begin{array}{l}20- \\
25 a\end{array}$ & $0,39 b$ & $10-25 a$ & Cundinamarca b & $\begin{array}{l}\text { a (CONIF, 2009); } \\
\text { b (Escobar, 1993) }\end{array}$ \\
\hline $\begin{array}{c}\text { Pinus } \\
\text { tecunumanii }\end{array}$ & $16 a$ & $0,51-0,56 b$ & $30-40 a$ & --- & $\begin{array}{l}\text { a (CONIF, 2009); } \\
\text { b (Barrance, 2004) }\end{array}$ \\
\hline $\begin{array}{l}\text { Schizolobium } \\
\text { parahibum }\end{array}$ & $16 a$ & $0,25-0,34 b$ & $13 a$ & --- & $\begin{array}{l}\text { a (CONIF, 2009); } \\
\text { b (Barrance, 2004) }\end{array}$ \\
\hline $\begin{array}{l}\text { Tabebuia } \\
\text { roseae }\end{array}$ & --- & $0,54 a$ & $8,4 b$ & $\begin{array}{l}\text { Arauca, } \\
\text { Córdoba, } \\
\text { Cundinamarca, } \\
\text { Huila, Tolima a }\end{array}$ & $\begin{array}{l}\text { a (Escobar, 1993); } \\
\text { b (Barrance, 2004) }\end{array}$ \\
\hline $\begin{array}{l}\text { Tectona } \\
\text { grandis }\end{array}$ & $\begin{array}{l}25- \\
28 a\end{array}$ & $0,53 b$ & $7-13 a$ & --- & $\begin{array}{l}\text { a (CONIF, 2009); } \\
\text { b (Escobar, 1993) }\end{array}$ \\
\hline
\end{tabular}




\section{Composición química}

La composición química del material lignocelulósico define las condiciones de pretratamiento, hidrólisis y fermentación de la materia prima. La composición química de la madera no puede definirse precisamente para una especie forestal dada, ni siquiera para un árbol dado. La composición química varía con la parte del árbol (raíz, tallo o rama), tipo de madera (por ejemplo, normal, tensión o compresión), ubicación geográfica, clima y condiciones del suelo. Los datos analíticos acumulados durante muchos años de trabajo y desde muchos laboratorios diferentes han ayudado a definir valores promedios esperados para la composición química de la madera. El análisis químico ordinario puede distinguir entre maderas duras (angiospermas) y maderas blandas (gimnospermas). Desafortunadamente, tales técnicas no pueden ser usadas para identificar especies forestales individuales, dada la variación dentro de cada especie y las similitudes entre muchas.

Existen dos componentes químicos importantes en la madera: Lignina (18-35\%) y carbohidratos (65-75\%). Ambos son materiales poliméricos complejos. Pequeñas cantidades de materiales extraños, la mayor parte en forma de extractivos orgánicos y de minerales inorgánicos (cenizas), también están presentes en la madera (normalmente 4-10\%). Globalmente, la madera tiene una composición elemental de $50 \%$ carbono, $6 \%$ hidrógeno, $44 \%$ oxígeno y trazas de diferentes iones metálicos.

La fracción de carbohidratos de la madera comprende celulosa y hemicelulosa. Los contenidos de celulosa varían de 40 a 50\% (base seca) y el contenido de hemicelulosa varía de 25 a 35\% (base seca).

No existe una compilación sistemática de la caracterización química de las maderas colombianas, salvo algunos esfuerzos por parte del SENA y de la Universidad Nacional (Maderas de Colombia) por generar bases de datos respecto a aspectos anatómicos y mecánicos de algunas especies nacionales, en el ámbito composicional solo es posible encontrar datos aislados para unas pocas especies maderables. Se hace necesario entonces recurrir a información foránea como aproximación a una valoración preliminar del factor composicional de algunas especies forestales. Realizado esto sobresalen inmediatamente algunos árboles dentro de los que cabe destacar las especies Pinus caribaea, Pinus patula, Schizolobium parahibum Eucalyptus camaldulensis y Pinus maximinoi por sus altos contenidos de celulosa $(48,06 \%, 48,75 \%, 49 \%$ y $52,38 \%$, respectivamente). Asimismo hay que resaltar los contenidos de lignina de las especies Cedrela odorata, Cupressus lusitánica, Pinus radiata, Eucalyptus camaldulensis y la especie Tectona grandis, con una presencia de lignina de 33\%, 33\%, 33,72\%, 34 y 35\%, respectivamente. A continuación en la Tabla 4 se muestra la composición química (celulosa, hemicelulosa y lignina) para algunas especies forestales de Colombia.

Si bien el contenido de azúcares fermentables y el contenido de lignina son los factores realmente importantes a la hora de valorar material lignocelulósico para producir etanol, esta información no se encuentra disponible de manera completa actualmente. Solo algunas especies maderables han sido caracterizadas en este aspecto, en investigaciones internacionales. Suecia es un estado pionero en producción de etanol a partir de material maderable, situación que ha sido el resultado de buen desarrollo científico con su material maderable de mayor disponibilidad: Las maderas blandas (Söderström, 2004). Picea sueca ha mostrado contenidos de glucano, manano, galactano xilano y arabinano de 53\%, 12\%, 2,1\%4,3\% y 0,9\% en base seca (Söderström, 2004). Algunos pinos han sido caracterizados de igual manera (Pinus banksiana, Pinus radiata, Pinus resinosa, Pinus rigida, Pinus strobus, Pinus sylvstris y Pinus taeda) reportando contenidos de glucano de 42-47\%, de xilano $6,0-9,3 \%$, de galactano de $1,4-3,1 \%$, de arabinano de $1,4-2,7 \%$ y de manano de $7,4-12 \%$ en base seca (Pettersen, 1984). Las materias duras también han gozado de poca investigación en el campo de los biocombustibles a pesar de sus contenidos de lignina relativamente más bajos y su mayor atacabilidad enzimática que las ubican en una buena posición en la escala de materiales con mayor factibilidad para producir etanol. En maderas duras se han desarrollado algunos estudios arrojando detalles de caracterización de 43\%, 15\%, 2,0\%, 1,2\% $3,2 \%$ en contenidos de glucano, xilano, galactano, arabinano y manano (Sassner, 2006). A pesar 
de que las pocas investigaciones llevadas a cabo han arrojado bajos porcentajes de conversión (Sassner, 2006; Söderström, 2004), la investigación con maderas locales es pertinente y más aún, la caracterización profunda de las especies forestales, con el objetivo de desarrollar nuevas perspectivas en el área de las energías alternativas.

Tabla 4: Composición química de algunas especies forestales

\begin{tabular}{ccccc}
\hline \hline Especie forestal & Celulosa (\%) & H-celulosa (\%) & Lignina (\%) & Referencias \\
\hline \hline Cedrela odorata & 37 & 18 & 33 & (Pettersen, 1984) \\
Cordia alliodora & 45 & 17 & 30 & (Pettersen, 1984) \\
Cupresus lusitánica & $39-40$ & 23 & $31-33$ & (Kropff, 2000) \\
Eucalyptus camaldulensis & $41-50$ & $14-19$ & $18-34$ & (Kropff, 2000) \\
Eucalyptus globulus & 45 & 23 & 27 & (Kropff, 2000) \\
Eucalyptus grandis & 41,2 & 28,3 & 23,2 & (Kropff, 2000) \\
Eucalyptus tereticornis & $45-48$ & $11-23$ & $22-30$ & (Kropff, 2000) \\
Eucalyptus urophylla & 19 & 24 & 17 & (Pettersen, 1984) \\
Gmelina arbórea & 47 & 20 & 29 & (Pettersen, 1984) \\
Pinus caribaea & 48,06 & 18,95 & 29,51 & (Mogollón, 2003) \\
Pinus kesiya & $37-43$ & $14-28$ & 28 & (Kropff, 2000) \\
Pinus maximinoi & 52,38 & --- & 28,22 & (Fonseca, 2006) \\
Pinus oocarpa & 42 & 23 & 29 & (Kropff, 2000) \\
Pinus patula & 48,75 & 32,75 & 18,5 & (Uribe, 1967) \\
Pinus radiata & 37,88 & 23,62 & 33,72 & (Berrocall, 2004) \\
Schizolobium parahibum & 49 & 14 & 26 & (Pettersen, 1984) \\
Tectona grandis & 33 & 40 & 35 & (Pettersen, 1984) \\
\hline \hline
\end{tabular}

\section{CONCLUSIONES}

El rendimiento o producción biomásica de una especie maderable es un factor importantísimo a la hora de evaluar la factibilidad de una especie arbórea para ser usada en la producción de etanol celulósico. Especies forestales disponibles en territorio colombiano como Eucalyptus camaldulensis, pinos Caribaea, Oocarpa y Tecunumanii y eucaliptos Grandis, Camaldulensis y Tereticornis tienen rendimientos volumétricos de biomasa lo suficientemente altos y turnos de crecimiento cortos como para garantizar un suministro rápido y constante de biomasa lignocelulósica para ser empleada como materia prima en un proceso de producción de biocombustibles.

Si bien las maderas blandas, encabezadas localmente por la especie $P$. patula, suponen una mayor resistencia a la liberación de los azúcares fermentables, el gran desarrollo que ostenta Colombia en la industria maderera con estas especies y su extensión en plantaciones comerciales tienen un efecto compensatorio.

En términos de composición química $P$. patula ofrece los mayores contenidos de celulosa y hemicelulosa con un contenido bajo de lignina y, por lo tanto, como una primera aproximación, la resistencia de material lignocelulósico proveniente de esta especie a la liberación se azúcares fermentables sería en un principio baja. Sin embargo, dadas las dificultades técnicas que presenta la biomasa maderable respecto a otros materiales lignocelulósicos como los pastos, y en particular, las maderas blandas respecto a las duras, es necesario llevar a cabo una caracterización detallada con materia prima local partiendo de la evaluación y las aseveraciones que acá se presentan.

Globalmente, las especies maderables $E$. camaldulensis y $P$. patula presentan valores favorables de composición química, condiciones edafológicas y rendimiento volumétrico lo que sugiere su susceptibilidad para ser empleadas en la producción de etanol carburante. La gran abundancia de este recurso maderero en Colombia en forma de plantaciones comerciales y su elevado uso en la industria maderera, son una buena fuente de abastecimiento de materia prima para la industria de los biocombustibles. 


\section{REFERENCIAS}

AGROSOFT, Pinus patula Schl. et Cham. Serie Especies Forestales. Reporte de especie $\mathrm{n}^{\circ} 2$ (2000). Medellín, Colombia,

http://www.oeidrustab.gob.mx/paquete_basico/med_amb/fichas_tecnicas/ficha_tecnica_pinus_pat _sp.PDF. Acceso: 25 de mayo (2010).

Bárcenas, G. y R. Dávalos, Importancia de la lignina en las contracciones de la madera: Revisión bibliográfica. Madera y Bosques: 5 (1), 13-26 (1999).

Barrance, A., J. Beer, D. Boshier, J. Chamberlain, J. Cordero, G. Detlefsen, B. Finegan, G. Galloway, M. Gómez, J. Gordon, M. Hands, J. Hellin, C. Hughes, M. Ibrahim, R. Leakey, F. Mesén, M. Montero, C. Rivas, E. Somarriba, y J. Stewart, Árboles de Centroamérica (2004). http://herbaria.plants.ox.ac.uk/adc/. Acceso: 25 de mayo (2010).

Berrocal, A., J. Baeza, J. Rodríguez, M. Espinosa y J. Freer, Effect of tree age on variation of Pinus radiate D. Don chemical composition. J. Chilean Chem. Soc.: 49 (3), 251-256 (2004).

Cinco, Y. y O. Mercado, Determinación de las propiedades físicas y de trabajabilidad del Eucalyptus camaldulensis. Copérnico: Revista arbitrada de divulgación científica: año I (2), 60-64 (2005).

Colombia virtual, Regiones económicas (2010), http://www.colombia-virtual.com/regioneseconomicas.html. Acceso: 25 de mayo (2010).

CONAFOR, Alnus acuminata H.B.K. SIRE - Paquetes tecnológicos (2010a), http://www.conafor.gob.mx/portal/docs/secciones/reforestacion/Fichas\%20Tecnicas/Alnus\%20acu minata.pdf. Acceso: 25 de mayo (2010).

CONAFOR, Pinus maximinoi H.E. Moore. SIRE - Paquetes tecnológicos (2010b). http://www.conafor.gob.mx/portal/docs/secciones/reforestacion/Fichas\%20Tecnicas/Pinus\%20max iminoi.pdf. Acceso: 25 de mayo (2010).

CONIF - Ministerio de Agricultura y Desarrollo Rural. Sector Forestal, Invierta en Colombia: 1 (1) (2009), http://www.inviertaencolombia.com.co/Adjuntos/089_Sector\%20Forestal\%202009-0813.pdf. Acceso: 25 de mayo (2010).

Duke, J., Eucalyptus grandis Hill ex Maiden. Center for New Crops \& Plant Products (1998a), http://www.hort.purdue.edu/newcrop/duke_energy/Eucalyptus_grandis.html\#Yields and Economics. Acceso: 25 de mayo (2010).

Duke, J., Eucalyptus tereticornis Sm. Center for New Crops \& Plant Products (1998b). http://www.hort.purdue.edu/newcrop/duke_energy/Eucalyptus_tereticornis.html. Acceso: 25 de mayo (2010).

Duke, J., Gmelina arborea Roxb. Center for New Crops \& Plant Products (1998c). http://www.hort.purdue.edu/newcrop/duke_energy/gmelina_arborea.html\#chemistry. Acceso: 25 de mayo (2010).

Escobar, Ó. y J. Rodríguez, Las Maderas en Colombia. Universidad Nacional de Colombia SENA. Medellín, Colombia. (1993).

Fonseca, M., Determinación de la composición química de la madera de Pino candelillo (Pinus maximinoi H. E. Moore) procedente de la fina Río Frío, Tactic, Alta Verapaz, Tesis de pregrado, Dpto. Ingeniería Química, Universidad de San Carlos. Guatemala (2006). 
Galbe, M. y Zacchi, G., Pretreatment of lignocellulosic materials for efficient bioethanol production. Adv. Biochem. Eng./Biotechnol. 108, 41-65. (2007).

Gifoni, V., Propiedades de chapas fabricadas con partículas de madeira de Eucalyptus Urophylla, Empresa Brasileira de Pesquisa Agropecuária (2004).

http://www.cenargen.embrapa.br/publica/trabalhos/cot111.pdf. Acceso: 25 de mayo (2010).

Gillespie, A., Pinus patula Schiede \& Deppe. Pino pátula, ocote. International Institute of Tropical Forestry (IITF). United States Department of Agriculture (USDA), Forest Service (2004). http://www.fs.fed.us/global/iitf/Pinuspatula.pdf. Acceso: 25 de mayo (2010).

Hamelinck, C.N., Van Hooijdonk, G. y Faaij, A.P.C. Ethanol from lignocellulosic biomass: technoeconomic performance in short-, middle- and long-term. Biomass Bioenergy (2005). 28 384-410.

ICRAF, International Center for Research in Agroforestry, Eucalyptus urophylla (1998), http://www.worldagroforestry.org/sea/Products/AFDbases/af/asp/SpeciesInfo.asp?SpID=821.

Acceso: 25 de mayo (2010).

Kropff, M., PROTA: Plant Resources of Tropical Africa (2000), http://database.prota.org/publishedspeciesEn.htm. Acceso: 25 de mayo (2010).

Maro, R., S. Chamshama, V. Nsolomo y S. Maliondo, Soil chemical characteristics in a natural forest and a Cupressus Lusitanica plantation at West Kilimanjaro, Northern Tanzania. J. Trop. Forest Sc.: 5 (4), 465-472 (1991).

Meskimen, G. y J. Francis, Eucalyptus grandis Hill ex Maiden. Eucalipto rosado. International Institute of Tropical Forestry (IITF). United States Department of Agriculture (USDA), Forest Service. http://www.fs.fed.us/global/iitf/Eucalyptusgrandis.pdf. Acceso: 25 de mayo (2010).

Ministerio de Agricultura y Desarrollo Rural. Resolución 711 del 31 de octubre de 1994. República de Colombia (2012). http://www.minagricultura.gov.co/02componentes/06com_03d_cif.aspx. Acceso: 10 de julio de 2012.

Mogollón, G., A. Aguilera e I. Gutiérrez, Caracterización del Pino Caribe resinado proveniente de plantaciones. Forest: 47 (2), $107-114$ (2003).

Naumann, R., B. Rocha, A. Oliveira, R. Della, J. Castro, A. Macêdo y A. Colli, Propriedades de chapas fabricadas com partículas de madeira de Eucalyptus urophylla S. T. Blake e de Schizolobium amazonicum Herb. Árvore: 32. (6), 1143-1150 (2003).

Pettersen, R., The chemical composition of wood: The chemistry of solid wood. Departamento de Agricultura de los Estados Unidos. Washington D.C. Estados Unidos (1984).

Proexport. Sector Forestal en Colombia. Colombia (2012).

Salazar, F., Cavazos, J., Martínez, J. L., Metodología basada en el Modelo de Referencia para Cadenas de Suministro para Analizar el Proceso de producción de Biodiesel a partir de Higuerilla. Información Tecnológica Vol. 23 Nº 1 (2012)

Sánchez, Ó., Cardona, C., Trends in biotechnological production of fuel ethanol from different feedstocks. Bioresour. Technol 99 5270-5295 (2008).

Sassner, P. Galbe, M. y Zacchi, G., Bioethanol production based on simultaneous saccharification and fermentation of steam-pretreated Salix at high dry-matter content. Enzyme and Microbial Technology 39, 756-762 (2006). 
Skolmen, R. y F. Ledig, F., Eucalyptus globulus Labill. Eucalipto Goma Azul. International Institute of Tropical Forestry (IITF). United States Department of Agriculture (USDA), Forest Service (2004). http://www.fs.fed.us/global/iitf/Eucalyptusglobulus.pdf. Acceso: 25 de mayo (2010).

Söderström, J., Galbe, M. y Zacchi, G., Effect of Washing on Yield in One- and Two-Step Steam Pretreatment of Softwood for Production of Ethanol. Biotechnol. Prog., Vol. 20, No. 3. (2004).

Sun, Y. y Cheng, J., Hydrolysis of lignocellulosic materials for ethanol production: A review, Bioresour. Technol. 83, 11. (2002).

Trujillo, E., Semillas Forestales Mejoradas para la Reforestación en Colombia. Revista M \& M: 48, 21-27 (2005).

Uribe, G., Pinus patula como base en la producción de pulpa para papel. Tesis de pregrado, Dpto. de Ingeniería Forestal, Universidad Nacional de Colombia. Medellín, Colombia (1967).

Varnero, M. T., Quiroz, M. S. y Álvarez, C. H., Utilización de Residuos Forestales Lignocelulósicos para Producción del Hongo Ostra (Pleurotus ostreatus). Información Tecnológica Vol. - 21 № 2, (2010).

Wishnie, M., D. Dent, E. Mariscal, J. Deago, N. Cedeño, D. Ibarra, R. Condit y P. Ashton, Initial performance and reforestation potential of 24 tropical tree species planted across a precipitation gradient in the Republic of Panama. Forest Ecol. and Manag.: 243, 39-49 (2007).

Zamora-Campos, E. y L. Mendizábal, Variación del peso específico de una prueba de procedencias/progenie de Pinus caribaea Mor. Var. Hondurensis Barr. y Golf. en la región de los Tuxtla, Veracruz, México. Foresta Veracruzana: 6 (2). $41-46$ (2004). 